\title{
Hereditary Hypophosphatemic rickets with Hypercalciuria: A case report and review of literature
}

\author{
Subal Ku.Pradhan ${ }^{1 *}$, Pawan Mutalik ${ }^{2}$, Sandeep Ku.Tripathy ${ }^{3}$, Arakhita Swain ${ }^{4}$ \\ Sardar Vallabh Bhai Patel Post Graduate Institute of Pediatrics (SVPPGIP) and SCB Medical College, \\ Cuttack, Odisha, India
}

\begin{abstract}
Hereditary hypophosphatemic rickets with Hypercalciuria (HHRH) is one of the four entities of hereditary hypophosphatemic rickets, characterized by vitamin D refractory rickets, Hyperphosphaturia, Hypercalciuria, hypophosphatemia, elevated serum 1, 25-dihydroxyvitamin D levels, normal serum Calcium, 25-hydroxyvitamin D and PTH levels. Clinically, it is difficult to differentiate the four entities without a genetic test. However, presence of Hypercalciuria by a simple bedside urine examination of spot calcium-creatinine ratio differentiates HHRH from other three entities of hereditary hypophosphatemic rickets. Correction of all the abnormalities, except for renal inorganic phosphate wasting can be achieved by simple prescription of oral phosphate supplementation. It is also the only type of refractory rickets in which Vitamin D supplementation is more hazardous than beneficial. In this study, we review the literature and describe a case of two year old female child who presented with vitamin D refractory rickets at our tertiary level pediatric centre in Cuttack, Odisha, India whose phenotypic characteristics corresponded with HHRH.
\end{abstract}

Keywords: refractory rickets, HHRH, urine calcium creatinine ratio, NPT2c gene, oral phosphate

\section{Case Report}

We report a case of a two year old first order female child born out of non-consanguineous marriage with delayed motor milestones. The child presented to us with the complaints of not able to walk without support, short height and not gaining weight adequately. The antenatal and natal history was uneventful with a birth weight of 3 kilograms $(\mathrm{kg})$ and length 49 centimeters $(\mathrm{cm})$. There was no history of polyuria, polydipsia or family history of similar complaints. Past history revealed that child had an episode of lower respiratory infection about 1 month back which was treated with oral antibiotics and anti-pyretics for 7 days. On examination, the child had features of active rickets (Radiograph as in Fig.l) with delayed dentition and normal systemic examination. Before referral, the child had received two doses of injectable Vitamin D (6, 00,000 IU) one week apart along with oral Calcium \& Vitamin D supplementation. On anthropometrical evaluation, the child was detected to have height $<3^{\mathrm{RD}}$ centile $(63 \mathrm{~cm})$ and weight $<5^{\mathrm{TH}}$ centile $(7 \mathrm{~kg})$ as per standard $\mathrm{WHO}$ growth charts. A clinical diagnosis of Vitamin D refractory Rickets was made and further work up was done which revealed $\mathrm{Hb}-10.2 \mathrm{gm} \%$, TLC- 8,600 cells $/ \mathrm{mm} 3$, Neutrophils-56\%, Eosinophils-4\%, Lymphocytes-39\%, Monocytes-1\%, Serum Calcium- $8.9 \mathrm{mg} / \mathrm{dl}$, Serum Phosphate- $2.9 \mathrm{mg} / \mathrm{dl}$, Serum Alkaline Phosphatase- $825 \mathrm{IU}$, Serum Free T3 levels- $1.16 \mathrm{nmol} / \mathrm{L}$, Serum Free T4 levels- $81.22 \mathrm{nmol} / \mathrm{L}$, Serum TSH levels- $5.98 \mu \mathrm{IU} / \mathrm{mL}$, Serum Parathyroid hormone (PTH) levels- $24.8 \mathrm{pg} / \mathrm{ml}$ (Normal- $14-72 \mathrm{pg} / \mathrm{ml}$ ), Serum 25-D levels- $56 \mathrm{ng} / \mathrm{mL}$ (Normal: 30-100 ng/ml), Serum 1,25-D levels- 54.6 IU/L (Normal: 19.6-54.3 IU/L), Tubular Reabsorption of Phosphate (TRP)- 76\% and Urine examination did not show proteinuria, ketone bodies, glycosuria or aminoaciduria.

Based on the above clinical features, resistance to vitamin D and Calcium supplementation and laboratory findings of hypophosphatemia, Hyperphosphaturia, normal serum calcium, PTH, 25- hydroxyvitamin $\mathrm{D}$ and high normal 1,25- dihydroxyvitamin D, a diagnosis of Hypophosphatemic Rickets was done. Clinically, all the four entities of hypophosphatemic rickets (XLH, ADHR, ARHR and HHRH) cannot be differentiated without a genetic test except evidence of Hypercalciuria in HHRH. Our case showed a Urinary spot Calcium Creatinine ratio of 1.56 and Ultrasonography of kidney showed both kidneys of normal size (Right Kidney size$77 \mathrm{~mm}$ x $33 \mathrm{~mm}$, Left Kidney size- $78 \mathrm{~mm}$ x $36 \mathrm{~mm}$ ) with bilateral medullary nephrocalcinosis. Based on the above investigations, a diagnosis of HHRH was done. Vitamin D and oral calcium supplementation was stopped and the patient was started immediately on oral phosphate supplementation at the dose of $500 \mathrm{mg}$ four times a day. In view of Hypercalciuria with nephrocalcinosis, the child was treated with short course of Chlorthiazide tablet $(25 \mathrm{mg}) \frac{1}{2}$ tablet once daily with dietary salt restriction. The child was followed up every three monthly for a period of one year and then 6 monthly subsequently. During each visit, monitoring of growth and titration of dosage of medicines with existing serum levels of phosphate, calcium, alkaline phosphatase and urine levels of calcium was done. 
At the end of two years of follow up, the child was able to walk and run without support with normal height and weight with evidence of complete healing of rickets as shown in Fig.2. The serum phosphate was normalized $(4.7 \mathrm{mg} / \mathrm{dl})$, serum calcium $8.8 \mathrm{mg} / \mathrm{dl}$, serum alkaline phosphate was $307 \mathrm{IU}$ and Urinary spot Calcium Creatinine ratio 0.14 as shown in Chart 1. Hypercalciuria significantly improved and Ultrasonography of kidney had no signs of nephrocalcinosis. Presently, the child is only on oral phosphate supplementation. She attends school regularly and participates in various sports and games.

\section{Discussion}

Rickets is a disorder of growing bone and characterized by accumulation of unminerilazed bone. It could be due to either a primary metabolic abnormalities of Calcium (Calcipenic rickets) or Phosphorus (Phosphopenic rickets) or a miscellaneous group associated with direct inhibitors of mineralization ${ }^{[1]}$. Phosphopenic or Hypophosphatemic rickets are mostly inherited, though they may rarely be acquired [tumorinduced osteomalacia (TIO), drug-induced Fanconi syndrome] ${ }^{[2]}$. Hypophosphatemic rickets are due to isolated renal phosphate wasting, caused by a mutant gene and clinically present as vitamin D refractory rickets. At least four different entities of hereditary hypophosphatemic rickets (XLH, ADHR, ARHR Type-1 \& Type-2, and HHRH) have been described as shown in table 1. They are classified based on the mode of inheritance and urinary excretion of calcium ${ }^{[3,4]}$. The PHEX, FGF-23, DMP-1, ENPP-1 and Na/Pi co-transporter (NPT-2c) genes have roles in renal phosphate absorption and maintaining serum Pi homeostasis ${ }^{[5,6,7,8,14]}$.

\section{PHEX gene:}

PHEX is a zinc $(\mathrm{Zn})$ metalloendopeptidase, the loss of which causes defective mineralization, hypophosphatemia and abnormal vitamin-D metabolism ${ }^{[9]}$. This gene is expressed in fully differentiated osteoblasts and the down regulation of its expression by PTH in vitro and in vivo is associated with inhibition of matrix mineralization ${ }^{[10]}$. The presence of inactivating PHEX mutations results in the enhanced renal phosphate transport inhibitory activity thus leading to hyperphosphatemia ${ }^{[11]}$. Out of all four entities, X-linked hypophosphatemic rickets (XLH) is the most common type of hereditary hypophosphatemic rickets and it occurs due to mutations in PHEX gene ${ }^{[12]}$.

\section{FGF-23 gene:}

FGF-23 reduces serum phosphate level by suppressing proximal tubular phosphate reabsorption and intestinal phosphate absorption. FGF-23 is produced by bone and acts on the kidney through a specific receptor system which is composed of Klotho and certain subtypes of FGF receptors. Excess actions of FGF-23 cause several hypophosphatemic diseases characterized by impaired renal phosphate reabsorption and rickets/osteomalacia (ADHR) ${ }^{[13,14]}$. In addition to being regulated by phosphate and vitamin $\mathrm{D}$, some clinical evidence suggests that FGF-23 production is regulated by PHEX, DMP-1, and ENPP1 genes, which encode distinct protein products, but the molecular mechanisms whereby FGF-23 is regulated by these factors are unknown $^{[4,13,15,16,17]}$. PHEX and FGF-23 genes also cause impaired synthesis of 1, 25-dihydroxyvitamin D by inhibitory actions on $1 \alpha$-hydroxylase enzyme.

\section{DMP-1 and ENPP-1 genes:}

Studies on Dmp1 mutations demonstrate that absence of DMP1 results in defective osteocyte maturation leading to pathological changes in bone mineralization manifesting as rickets and osteomalacia with isolated renal phosphate-wasting associated with elevated FGF-23 levels and normocalciuria (ARHR) ${ }^{[6]}$. ENPP1 gene causes ARHR with phosphaturia by positional cloning and also via an unreported mechanism which involves the common FGF-23 pathway ${ }^{[14]}$.

\section{NPT-2c gene:}

$\mathrm{Na}$-dependent $\mathrm{Pi}(\mathrm{Na} / \mathrm{Pi})$ transporters in the brush border membrane of proximal tubular cells mediate the rate-limiting step in the overall Pi-reabsorptive process and $\mathrm{Na} / \mathrm{Pi}$ co transport. Several studies suggest that type IIc co transporter in $\mathrm{Pi}$ reabsorption plays a major role in $\mathrm{HHRH}$. The type IIc $\mathrm{Na} / \mathrm{Pi}$ transporter is regulated by parathyroid hormone, dietary $\mathrm{Pi}$, and fibroblast growth factor 23 , and studies suggest a differential regulation of the IIa and IIc transporters ${ }^{[18]}$.

HHRH is a rare condition and only a few sporadic cases have been reported in literature. The first case of HHRH was described in a Bedouin tribe in 1985. It is inherited as a completely penetrating autosomal recessive trait and the primary defect is a phosphate leak in urine ${ }^{[19]}$. The resultant hypophosphatemia caused appropriate elevation of 1, 25-dihydroxyvitamin D, increased calcium absorption, suppression of parathyroid, and Hypercalciuria. Also phosphate deficiency is a potent and direct stimulus to renal 25-dihydroxyvitamin D-1alpha-hydroxylase activity ${ }^{[20]}$. This process is independent of Parathyroid hormone ${ }^{[21]}$. In contrast to XLH, ADHR \& ARHR (low or relative deficient 1,25-dihydroxyvitamin D level); HHRH is characterized by elevated 
serum levels of 1, 25-dihydroxyvitamin D for low serum Pi levels despite suppressed PTH and this is due to NPT-2c gene mutations. Hypophosphatemia caused by both PHEX and FGF23 mutations affects not only renal phosphate reabsorption but also the synthesis of 1,25-dihyroxyvitamin $\mathrm{D}$ due to the lack of up regulation of 25hydroxyvitamin D 1 $\alpha$-hydroxylase. This up-regulation in response to low serum phosphate is intact in HHRH, which further argues for a primary defect in renal phosphate reabsorption in this condition ${ }^{[8]}$.

The accurate diagnosis of HHRH and distinction with other form of hypophosphatemic rickets has important therapeutic implications. Unlike other entities of hereditary hypophosphatemic rickets which require administration of active vitamin D along with phosphate, HHRH is treated with only phosphate supplementation. The addition of vitamin D can worsen the Hypercalciuria, increase intestinal absorption of calcium and can create complications such as nephrocalcinosis and renal damage ${ }^{[22]}$.

\section{Treatment \& prognosis:}

Sole therapy with oral phosphate supplements resolves or improves hypophosphatemia, Hypercalciuria, and prevents progression of skeletal abnormalities without increasing PTH levels. In the absence of other biomarkers, 1,25-dihyroxyvitamin D levels may be a helpful laboratory parameter for assessing the treatment efficacy and compliance ${ }^{[23]}$. Therapy with vitamin D analogues must be avoided ${ }^{[24,25]}$.

\section{Teaching Points}

1. All the four entities of hereditary hypophosphatemic rickets clinically present as Vitamin D refractory rickets.

2. The clinical manifestations of XLH, ADHR and ARHR are related to dysregulation of FGF-23 along with impaired production of 1,25 dihydroxyvitamin $\mathrm{D}$ and normal urinary calcium levels.

3. The clinical manifestations of HHRH are due to mutation of NPT-2c gene resulting in elevation of 1,25dihydroxyvitamin D and Hypercalciuria.

\section{References}

[1]. Imel EA, Carpenter TO. Rickets: The skeletal disorders of impaired calcium or phosphate availability. Pediatr Endocrinol. 2013; 2: 357-378.

[2]. Jagtap VS, Sarathi V, Lila AR, Bandgar T, Menon P, Shah NS. Hypophosphatemic rickets. Indian J Endocrinol Metab. 2012 Mar; 16(2):177-82. Doi: 10.4103/2230-8210.93733.

[3]. Alizadeh Naderi AS, Reilly RF. Hereditary disorders of renal phosphate wasting. Nat Rev Nephrol2010;6:65765.10.1038/nrneph.2010.121.

[4]. Carpenter TO. The expanding family of hypophosphatemic syndromes. J Bone Miner Metab2012;30:1-9.10.1007/s00774-011-0340-2.

[5]. The ADHR Consortium Autosomal dominant Hypophosphataemic rickets is associated with mutations in FGF23. Nat Genet 2000;26:345-8.10.1038/81664

[6]. Feng JQ., Ward LM., Liu S., Lu Y., Xie Y., Yuan B., et al. Loss of DMP1 causes rickets and osteomalacia and identifies a role for osteocytes in mineral metabolism. Nat Genet 2006;38:1310-5.10.1038/ng1905.

[7]. Rutsch F., Ruf N., Vaingankar S., Toliat MR., Suk A., Höhne W., et al. Mutations in ENPP1 are associated with 'idiopathic' infantile arterial calcification. Nat Genet 2003;34:379-81.10.1038/ng1221.

[8]. Lorenz-Depiereux B., Benet-Pages A., Eckstein G., Tenenbaum-Rakover Y., Wagenstaller J., Tiosano D., et al. Hereditary Hypophosphatemic Rickets with Hypercalciuria is caused by Mutations in the Sodium-Phosphate Co transporter Gene SLC34A3. Am J Hum Genet 2006;78:193-201.10.1086/499410.

[9]. Rowe PSN. The wrickkened-pathways of FGF23, MEPE and PHEX. Crit Rev Oral Biol Med.2004; 15(5):264-281

[10]. Alos N, Ecarot B. Down regulation of osteoblast Phex expression by PTH. Bone. 2005 Oct; 37(4):589-98

[11]. Nesbitt T, Fujiwara I, Thomas R, Xiao ZS, Quarles LD, Drezner MK. Coordinated maturational regulation of PHEX and renal phosphate transport inhibitory activity: evidence for the pathophysiological role of PHEX in X-linked hypophosphatemia. J Bone Miner Res. 1999 Dec; 14(12):2027-35.

[12]. A gene (PEX) with homologies to endopeptidases is mutated in patients with X-linked hypophosphatemic rickets. The HYP Consortium. Nat Genet. 1995 Oct; 11(2):130-6.

[13]. Fukumoto S. Physiological regulation and disorders of phosphate metabolism--pivotal role of fibroblast growth factor 23. Intern Med. 2008; 47(5):337-43. Epub 2008 Mar 3.

[14]. Levy-Litan V, Hershkovitz E, Avizov L, Leventhal N, Bercovich D et al. Autosomal-recessive hypophosphatemic rickets is associated with an inactivation mutation in the ENPP1 gene. Am J Hum Genet. 2010 Feb 12; 86(2):273-8. Doi: 10.1016/j.ajhg.2010.01.010. Epub 2010 Feb 4.

[15]. Alon US. Clinical practice. Fibroblast growth factor (FGF)23: a new hormone. Eur J Pediatr. 2011; 170:545-554.

[16]. Gattineni J, Baum M. Genetic disorders of phosphate regulation. Pediatr Nephrol. 2012; 27:1477-1487.

[17]. Baroncelli GI, Toschi B, Bertelloni S. Hypophosphatemic rickets. Curr Opin Endocrinol Diabetes Obes. 2012; $19: 460-467$.

[18]. Miyamoto K, Ito M, Tatsumi S, Kuwahata M, Segawa H. New aspect of renal phosphate reabsorption: the type IIc sodium-dependent phosphate transporter. Am J Nephrol. 2007; 27(5):503-15. Epub 2007 Aug 7.

[19]. Tieder M, Modai D, Samuel R, Arie R, Halabe A, Bab I et al. Hereditary hypophosphatemic rickets with Hypercalciuria. N Engl J Med. 1985 Mar 7; 312(10):611-7.

[20]. Haussler M, Hughes M, Baylink D, Littledike ET, Cork D, Pitt M. Influence of phosphate depletion on the biosynthesis and circulating level of 1alpha, 25-dihydroxyvitamin D. Adv Exp Med Biol. 1977; 81:233-50.

[21]. Kumar R. Metabolism of 1, 25-dihydroxyvitamin D3. Physiol Rev. 1984 Apr; 64(2):478-504.

[22]. Glorieux FH. Rickets, the continuing challenge. N Engl J Med 1991, 325: 1875-1877.

[23]. Yu Y, Sanderson SR, Reyes M, Sharma A, Dunbar N, Srivastava T et al. Novel NaPi-IIc mutations causing HHRH and idiopathic hypercalciuria in several unrelated families: long-term follow-up in one kindred. Bone. 2012 May; 50(5):1100-6. Doi: 10.1016/j.bone.2012.02.015. Epub 2012 Feb 24. 
[24]. Bagga A, Hari P, Vasudev AS, Sharma A, Srivastava RN.Hypophosphatemic rickets with Hypercalciuria. Indian Pediatr. 1995 Nov; 32(11):1210-4.

[25]. Lucas MN, Dias S. A case of hereditary Hypophosphataemic rickets with Hypercalciuria (HHRH). Sri Lanka Journal of Child Health, $2006 ; 35: 141-3$.

\section{FIGURES LEGENDS}

Fig.1: Radiograph at presentation showing features of active rickets like cupping, fraying and widening of lower end of femur and upper end of tibia in both the lower limbs

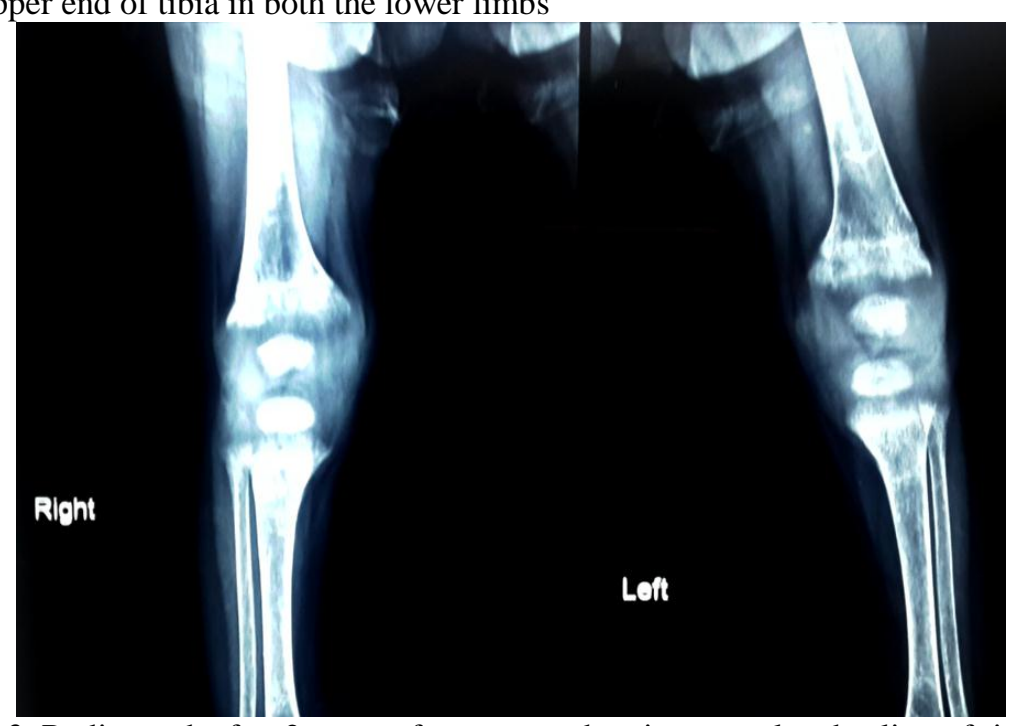

Fig.2: Radiograph after 2 years of treatment showing complete healing of rickets

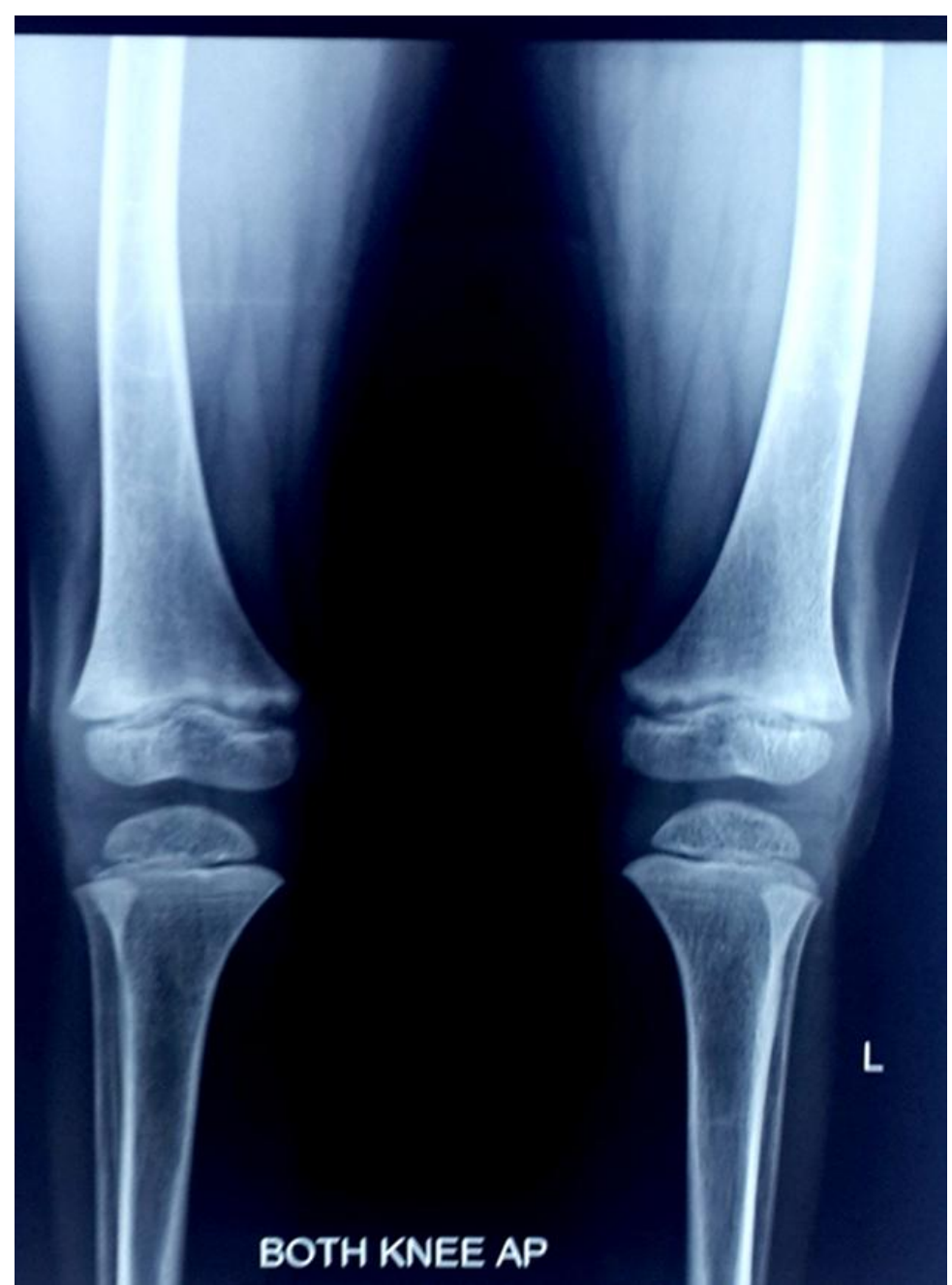


TABLE-1

Differentiation of Hereditary hypophosphatemic rickets

\begin{tabular}{|lllllllllll|}
\hline Disorder & FGF-23 & $\mathbf{C a}$ & $\mathbf{P i}$ & $\mathbf{A L P}$ & $\mathbf{P T H}$ & $\mathbf{2 5}-\mathrm{D}$ & $\mathbf{1 , 2 5}-\mathrm{D}$ & $\begin{array}{l}\text { Urine } \\
\text { Ca }\end{array}$ & $\begin{array}{l}\text { Urine } \\
\text { Pi }\end{array}$ & Mutation \\
\hline XLH & $\uparrow / \mathrm{RD}$ & $\mathrm{N}$ & $\downarrow$ & $\uparrow$ & $\mathrm{N}$ & $\mathrm{N}$ & $\downarrow / \mathrm{RD}$ & $\mathrm{N}$ & $\uparrow$ & PHEX \\
ADHR & $\uparrow / \mathrm{RD}$ & $\mathrm{N}$ & $\downarrow$ & $\uparrow$ & $\mathrm{N}$ & $\mathrm{N}$ & $\downarrow / \mathrm{RD}$ & $\mathrm{N}$ & $\uparrow$ & FGF-23 \\
ARHR & $\uparrow / \mathrm{RD}$ & $\mathrm{N}$ & $\downarrow$ & $\uparrow$ & $\mathrm{N}$ & $\mathrm{N}$ & $\downarrow / \mathrm{RD}$ & $\mathrm{N}$ & $\uparrow$ & DMP-1 \\
Type 1 & & & & & & & & & & \\
ARHR & $\uparrow / \mathrm{RD}$ & $\mathrm{N}$ & $\downarrow$ & $\uparrow$ & $\mathrm{N}$ & $\mathrm{N}$ & $\downarrow / \mathrm{RD}$ & $\mathrm{N}$ & $\uparrow$ & ENPP-1 \\
Type 2 & $\downarrow$ & $\mathrm{N}$ & $\downarrow$ & $\uparrow$ & $\mathrm{N} \downarrow$ & $\mathrm{N}$ & $\uparrow$ & $\uparrow$ & $\uparrow$ & $\mathrm{NPT} 2 \mathrm{c}$ \\
HHRH & $\downarrow$ & & & & & & & & & \\
\hline
\end{tabular}

RD- Relatively deficient

N- Normal

Pi- Inorganic phosphate

XLH- X linked hypophosphatemic rickets

ADHR- Autosomal dominant hypophosphatemic rickets

ARHR-Autosomal recessive hypophosphatemic rickets

HHRH- Hereditary Hypophosphatemic rickets with Hypercalciuria

Ca- Calcium

ALP- Alkaline phosphatase

PTH- Parathyroid hormone

PHEX-phosphate-regulating gene with homologies to endopeptidases on the X chromosome

FGF- Fibroblast growth factor

DMP- Dentin matrix acidic phosphoprotein

ENPP- Ectophosphodiesterase/nucleotide phosphohydrolase

NPT2c- Na (+)-Dependent Phosphate Co transporter 2C

\section{CHART 1}

Resolution of hypercalciuria and normalization of serum phosphorus on follow-up

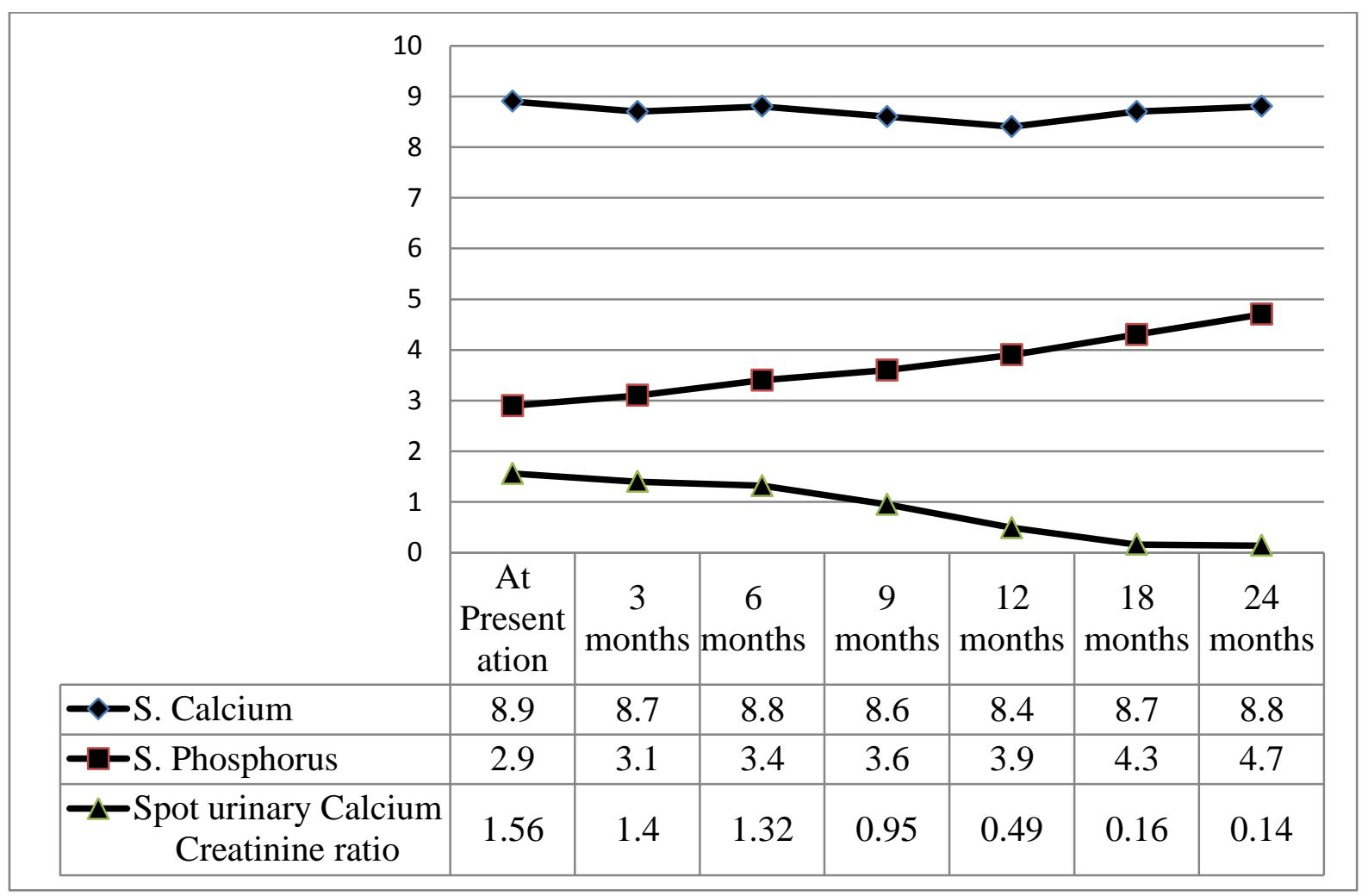

